TITLE:

\title{
A Study on the Spectrum of the Sampled-Data Transfer Operator with Application to Robust Exponential Stability Problems
}

\section{AUTHOR(S):}

Hagiwara, Tomomichi

\section{CITATION:}

Hagiwara, Tomomichi. A Study on the Spectrum of the Sampled-Data Transfer Operator with Application to Robust Exponential Stability Problems. SIAM Journal on Control and Optimization 2005, 44(1): 313-327

ISSUE DATE:

2005-08-22

URL:

http://hdl.handle.net/2433/194120

RIGHT:

(c) 2005 Society for Industrial and Applied Mathematics 


\title{
A STUDY ON THE SPECTRUM OF THE SAMPLED-DATA TRANSFER OPERATOR WITH APPLICATION TO ROBUST EXPONENTIAL STABILITY PROBLEMS*
}

\author{
TOMOMICHI HAGIWARA
}

\begin{abstract}
This paper begins by studying some spectral properties of the transfer operators of sampled-data systems described by applying the lifting technique. Through a "nonasymptotic" characterization of the transfer operator, its spectrum is determined in terms of finite-dimensional eigenvalue problems. Then, it is shown that a close connection with such eigenvalue problems and the exponential stability condition can be exploited to study the robust internal (exponential) stability problem of sampled-data systems. Since the transfer operator is relevant to input-output characteristics, the relationship between input-output stability and internal stability is also discussed in the context of sampled-data systems.
\end{abstract}

Key words. sampled-data system, spectral analysis, robust stability, $L_{2}$-stability, exponential stability

AMS subject classifications. 47A10, 47N70, 93C57, 93D09, 93D20

DOI. $10.1137 / \mathrm{S} 036301290343682 \mathrm{X}$

1. Introduction. The widespread use of digital controllers has stimulated the study of sampled-data systems with their intersample behavior taken into account, and a lot of important results have been obtained since the late 1980s. Among them are the studies on the $H_{\infty}$ control problem $[1,2,3,4]$ and robust stability problem $[5,6,7,8,9]$ of sampled-data systems, as well as the continuous-time lifting technique $[1,2,10]$ and the frequency response theory $[11,12]$. In the studies of the $H_{\infty}$ control and robust stability problems, $L_{2}$-stability [13], $L_{2}$-induced norm [14], and $H_{\infty}$ norm [15] of sampled-data systems play important roles, and these notions can be dealt with also in the frequency domain with the transfer operator $[1,2,10,11,15]$ of sampleddata systems. Also, another frequency-domain study has been conducted in $[16,17]$ by introducing the notion of positive-real sampled-data systems, and some phase properties of sampled-data systems were discussed. This study has been extended in $[18,19]$, which lead to the positive-realness approach (or the passivity approach) to the robust stability analysis of sampled-data systems. Transfer operators play an important role also in such an approach.

Thus, it is important to study the properties of the transfer operators so that the scope of the frequency-domain studies of sampled-data systems can be extended further. In this paper, we focus on the spectrum of transfer operators, and clarify some useful spectral properties. More specifically, we show that the spectrum of the transfer operator can be characterized by means of finite-dimensional eigenvalue problems. Then, it is demonstrated that such spectral analysis is indeed useful in the study of sampled-data systems by applying it to the robust internal (exponential) stability analysis of sampled-data systems. Furthermore, since the transfer operator is relevant

\footnotetext{
* Received by the editors October 26, 2003; accepted for publication (in revised form) January 15, 2005; published electronically August 22, 2005.

http://www.siam.org/journals/sicon/44-1/43682.html

${ }^{\dagger}$ Department of Electrical Engineering, Kyoto University, Kyotodaigaku-Katsura, Nishikyo-ku, Kyoto 615-8510, Japan (hagiwara@kuee.kyoto-u.ac.jp).
} 
to the input-output characteristics, we apply the results of the spectral analysis to relate (robust) $L_{2}$-stability and (robust) internal stability in the context of sampleddata systems. The consequent result is not surprising, but to the best knowledge of the author, studies relating robust $L_{2}$-stability and robust internal stability in the context of sampled-data systems are rare, with the study by the author and a colleague in [9] being an exception. Nevertheless, that study in [9] is limited to the case of additive and multiplicative perturbations, unfortunately, and it is quite hard to extend it to the general case. The present paper shows that our spectral analysis provides a simple and rigorous proof to relate these two stability notions. Here, it would be worth mentioning that a similar problem of relating $L_{2}$-stability and exponential stability has been studied, e.g., in $[20,21]$ for a class of (ordinary type of) infinite-dimensional systems, but those studies do not cover the present setting nor do our developments here follow similar techniques to those employed therein.

The contents of this paper are as follows. In section 2, we review the notion of the transfer operator $\widehat{G}(z)$ of sampled-data systems with a slight but crucial extension (i.e., its nonasymptotic characterization). This characterization allows us to introduce some appropriate nonzero initial states to the study of the mapping defined by the transfer operator, and makes it fairly easy to carry out the following discussions (e.g., the derivations of Theorem 5 and Proposition 7 ). In section 3, we study the spectral properties of the transfer operators, and show that they are nearly as amenable as those of compact normal operators, even though the transfer operators are generally noncompact and nonnormal. Based on these properties, we further show that the spectrum of the transfer operator $\widehat{G}(z)$ can be characterized with finite-dimensional eigenvalue problems for each $z$ such that $\widehat{G}(z)$ is well-defined. Section 4 applies the spectral study in section 3 to the study of robust internal (exponential) stability of sampled-data systems against perturbations. More specifically, subsection 4.1 studies the case where the perturbations are identities up to a real scalar constant, and the basic result for this case is applied in subsection 4.2 to the study of robust internal stability of sampled-data systems with general perturbations. In particular, we give a rigorous proof to the equivalence of robust $L_{2}$-stability and robust internal stability when the nominal sampled-data system and perturbations are internally stable; roughly speaking, we show that whatever robust internal stability/robust performance problems we may consider in the sampled-data setting, the conditions in the $L_{2}$-stability context are enough to guarantee the robust stability/performance in the internal stability context, provided that the perturbations belong to the class of internally stable finite-dimensional LTI (linear time-invariant) or $h$-periodic systems (where $h$ is the sampling period). Section 5 concludes the paper with some remarks.

We use the following notation in this paper: $\lambda(\cdot)$ denotes the set of the eigenvalues of a finite-dimensional matrix, while $\sigma(\cdot)$ denotes the spectrum of an operator. $\sigma_{l e}(\cdot)$, $\sigma_{r e}(\cdot)$, and $\sigma_{e}(\cdot)$ denote the left essential, right essential, and essential spectrum, respectively, [22]. Furthermore, whenever we refer to internal stability in what follows, it means exponential stability.

2. Transfer operators of sampled-data systems. In this paper, we deal with the sampled-data system $\Sigma_{0}$ shown in Figure 1 , where $P$ denotes the continuoustime generalized plant, $\Psi$ the discrete-time controller, $\mathcal{H}$ the zero-order hold, and $\mathcal{S}$ the ideal sampler. Solid lines represent continuous-time (vector) signals, while dashed lines discrete-time (vector) signals. The underlying sampling period will be denoted by $h$. We assume that the state-space representations of $P$ and $\Psi$ are given, 


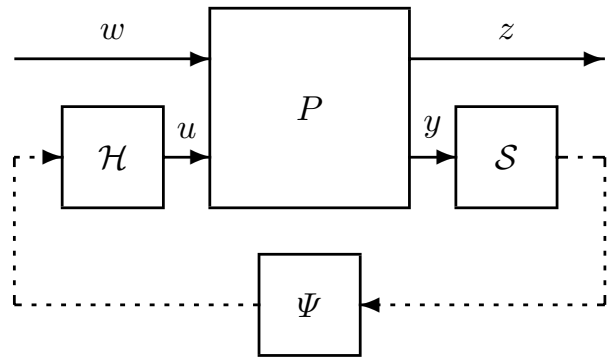

FiG. 1. Open-loop sampled-data system $\Sigma_{0}$.

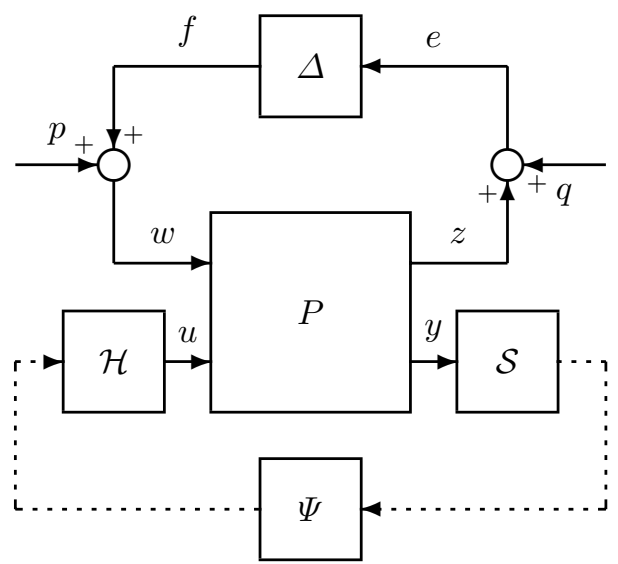

FiG. 2. Closed-loop sampled-data system $\Sigma_{\Delta}$.

respectively, by

$$
\begin{aligned}
\frac{d x}{d t} & =A x+B_{1} w+B_{2} u \\
z & =C_{1} x+D_{11} w+D_{12} u \\
y & =C_{2} x
\end{aligned}
$$

and

$$
\begin{aligned}
\xi_{k+1} & =A_{\Psi} \xi_{k}+B_{\Psi} y_{k} \\
u_{k} & =C_{\Psi} \xi_{k}+D_{\Psi} y_{k},
\end{aligned}
$$

where $y_{k}=y(k h)$ and $u(t)=u_{k}(k h \leq t<(k+1) h)$. In the following arguments, we assume that $\Sigma_{0}$ is internally (exponentially) stable [13, 23]. For lack of better terminologies, we call $\Sigma_{0}$ an open-loop sampled-data system, while if $w$ is given as $w=\Delta z$ with some causal mapping $\Delta$, then we call the resulting system a closed-loop sampled-data system, which we denote by $\Sigma_{\Delta}$. Also, the corresponding input-output mapping from $\left[p^{T}, q^{T}\right]^{T}$ to $\left[f^{T}, z^{T}\right]^{T}$ in Figure 2 will be denoted by $\mathcal{G}_{\Delta}$ in the following, when it is well-defined. If $\mathcal{G}_{\Delta}$ maps $L_{2}$ into $L_{2}$, and if its $L_{2}$-induced norm is bounded, then $\mathcal{G}_{\Delta}$ is said to be $L_{2}$-stable. In subsection 2.1, we review the lifted description and transfer operator $[1,2,10,11,15]$ of the open-loop sampled-data system $\Sigma_{0}$. Then, in subsection 2.2 , a slightly different interpretation of the transfer operators of sampled-data systems is given. 
2.1. Lifted description and transfer operators of sampled-data systems. With a slight abuse of notation, the Hilbert space of square integrable vector functions over the time interval $[0, h)$ with the standard inner product will be denoted by $\mathcal{K}$, whatever the dimension of the vector may be. The Euclidean space with dimension $\operatorname{dim}(x)$ will be denoted by $\mathcal{F}_{x}$. We also define $\mathcal{F}_{u}$ and $\mathcal{F}_{\xi}$ in a similar way, and we further define $\mathcal{F}:=\mathcal{F}_{x} \oplus \mathcal{F}_{\xi}$. Now, introduce the following matrices $A_{d}, B_{d 2}$, and $C_{d 2}$, and the operators $\mathbf{B}_{1}, \mathbf{C}_{1}, \mathbf{D}_{11}$, and $\mathbf{D}_{12}$ :

$$
\begin{gathered}
A_{d}:=\exp (A h), \quad B_{d 2}:=\int_{0}^{h} \exp (A \sigma) B_{2} d \sigma, \quad C_{d 2}:=C_{2} \\
\mathbf{B}_{1}: \mathcal{K} \ni w \mapsto \int_{0}^{h} \exp (A(h-\sigma)) B_{1} w(\sigma) d \sigma \in \mathcal{F}_{x} \\
\mathbf{C}_{1}: \mathcal{F}_{x} \ni x \mapsto z \in \mathcal{K}, \quad z(\theta)=C_{1} \exp (A \theta) x \\
\mathbf{D}_{11}: \mathcal{K} \ni w \mapsto z \in \mathcal{K}, \quad z(\theta)=\int_{0}^{\theta} C_{1} \exp (A(\theta-\sigma)) B_{1} w(\sigma) d \sigma+D_{11} w(\theta) \\
\mathbf{D}_{12}: \mathcal{F}_{u} \ni u \mapsto z \in \mathcal{K}, \quad z(\theta)=\int_{0}^{\theta} C_{1} \exp (A(\theta-\sigma)) B_{2} d \sigma u+D_{12} u .
\end{gathered}
$$

Then, the lifted description of the sampled-data system $\Sigma_{0}$ is given by

$$
\chi_{k+1}=\mathcal{A} \chi_{k}+\mathcal{B} \widehat{w}_{k}, \quad \widehat{z}_{k}=\mathcal{C} \chi_{k}+\mathcal{D} \widehat{w}_{k},
$$

where $\chi_{k}:=\left[x(k h)^{T}, \xi_{k}^{T}\right]^{T}$, and the associated transfer operator $\widehat{G}(z)$ is defined by

$$
\widehat{G}(z):=\mathcal{C}(z I-\mathcal{A})^{-1} \mathcal{B}+\mathcal{D},
$$

where

$$
\begin{gathered}
\mathcal{A}:=\left[\begin{array}{cc}
A_{d}+B_{d 2} D_{\Psi} C_{d 2} & B_{d 2} C_{\Psi} \\
B_{\Psi} C_{d 2} & A_{\Psi}
\end{array}\right]: \mathcal{F} \rightarrow \mathcal{F}, \quad \mathcal{B}:=\left[\begin{array}{c}
\mathbf{B}_{1} \\
0
\end{array}\right]: \mathcal{K} \rightarrow \mathcal{F} \\
\mathcal{C}:=\left[\begin{array}{ll}
\mathbf{C}_{1} & \mathbf{D}_{12}
\end{array}\right]\left[\begin{array}{cc}
I & 0 \\
D_{\Psi} C_{d 2} & C_{\Psi}
\end{array}\right]: \mathcal{F} \rightarrow \mathcal{K}, \quad \mathcal{D}:=\mathbf{D}_{11}: \mathcal{K} \rightarrow \mathcal{K} .
\end{gathered}
$$

In (8) above, $\widehat{w}$ and $\widehat{z}$ denote, respectively, the lifted representations of $w$ and $z$ (see the subsequent subsection for details). Note that $\mathcal{A}$ is a finite-dimensional matrix, and that $\widehat{G}(z)$ takes a value on the class of linear bounded operators on $\mathcal{K}$ for each $z$ unless $z$ is an eigenvalue of $\mathcal{A}$. The importance of $\widehat{G}(z)$ lies in that it captures all the intersample behavior (i.e., the aliasing phenomena) in $\Sigma_{0}[1,2,10,15,11]$.

In the following, we assume $\operatorname{dim}(w)=\operatorname{dim}(z)$ so that $D_{11}$ is square, unless otherwise stated explicitly. Also, with a slight abuse of notation ${ }^{1}$, the operator of multiplication by the matrix $D_{11}$ that maps $w(\cdot) \in \mathcal{K}$ to $z(\cdot)=D_{11} w(\cdot) \in \mathcal{K}$ is also denoted by $D_{11}$. Then, the operator $\mathbf{D}_{11}$ given in (6), known as the compression operator, can be rewritten as $\mathbf{D}_{11}=\mathbf{D}_{110}+D_{11}$ with an obvious definition of $\mathbf{D}_{110}$, and accordingly, $\mathcal{D}$ can also be rewritten as $\mathcal{D}=\mathcal{D}_{0}+D_{11}$. Then, $\mathcal{D}_{0}$ is compact, so that $\mathcal{D}$ (and thus $\widehat{G}(z))$ is compact if and only if $D_{11}=0$ (see, e.g., [11]).

\footnotetext{
${ }^{1}$ It will be clear from the context whether $D_{11}$ refers to the operator of multiplication or the underlying matrix. However, it would be worthwhile mentioning that whenever we refer to $\sigma_{l e}\left(D_{11}\right)$, $\sigma_{r e}\left(D_{11}\right)$, and $\sigma_{e}\left(D_{11}\right)$, we are talking about $D_{11}$ viewed as an operator, because otherwise these spectra are always empty.
} 
2.2. Nonasymptotic input-output relations about EMP-signals. In this subsection, we aim at giving a "nonasymptotic" characterization of the transfer operator $\widehat{G}(z)$. That interpretation is not surprising and only a slight modification of the well known "asymptotic" interpretation of the transfer operator, and is largely a review of the preliminary part of the arguments in [24], but does play an important role in the subsequent arguments. As such, we review somewhat detailed descriptions for this interpretation. To this end, let us begin by reviewing the lifting technique used in the derivation of the lifted description of sampled-data systems. Given a (vector) signal $w$ over the nonnegative time interval $[0, \infty)$, the lifting operation of $w$ is defined as

$$
w \mapsto\left\{\widehat{w}_{k}\right\}_{k=0}^{\infty},
$$

where $\widehat{w}_{k}$ is given by

$$
\widehat{w}_{k}(\theta)=w(k h+\theta) \quad(0 \leq \theta<h, k=0,1,2, \cdots) .
$$

The signal $w$ is called an EMP-signal of characteristic multiplier $\zeta$ [25] if its lifted representation satisfies

$$
\widehat{w}_{k}(\theta)=\widehat{w}_{0}(\theta) \zeta^{k} \quad(0 \leq \theta<h)
$$

for some $\widehat{w}_{0} \in \mathcal{K}$ and a complex number $\zeta$, where EMP stands for "exponentially modulated periodic." In this case, let us denote the "initial function" $\widehat{w}_{0}$ of the EMPsignal $w$ by $\widehat{w}_{0}=\operatorname{INI}(w)$. Conversely, let us denote by $w=\operatorname{EMP}\left(\widehat{w}_{0}\right)$ the operation of constructing an EMP-signal $w$ from the initial function $\widehat{w}_{0}$ according to (13) and then (12). Note in these notations that we suppress the underlying characteristic multiplier $\zeta$ for simplicity, and that $\operatorname{INI}(w)(\theta)$ is nothing but $w(\theta)$ for $0 \leq \theta<h$.

It is a fact [11] that the output $z$ of $\Sigma_{0}$ to the EMP-signal $w=\operatorname{EMP}\left(\widehat{w}_{0}\right)$ with characteristic multiplier $\zeta \notin \lambda(\mathcal{A})$ tends to some EMP-signal $z^{\star}$ of the same characteristic multiplier $\zeta$ and that the initial function $\widehat{z}_{0}=\operatorname{INI}\left(z^{\star}\right)$ of the asymptotic response $z^{\star}$ is given by

$$
\widehat{z}_{0}=\widehat{G}(\zeta) \widehat{w}_{0} .
$$

Note carefully that $\widehat{z}_{0}(\theta)(0 \leq \theta<h)$ in (14) is generally different from the actual response $z(t)(0 \leq t<h)$ of $\Sigma_{0}$ for the zero initial state (given by $z=\mathcal{D} \widehat{w}_{0}$ ), because the actual response $z$ is not exactly an EMP-signal over the entire nonnegative time interval but it just tends to the EMP signal $z^{\star}$ as $t$ goes to infinity.

However, given any $\widehat{w}_{0} \in \mathcal{K}$, let us take $\widehat{z}_{0}$ given by (14) for $\zeta \notin \lambda(\mathcal{A})$, and let us construct the EMP-signals $w=\operatorname{EMP}\left(\widehat{w}_{0}\right)$ and $z=\operatorname{EMP}\left(\widehat{z}_{0}\right)$ with characteristic multiplier $\zeta$. Then, it is easy to show that there exists an appropriate initial state $\chi_{0}$ of $\Sigma_{0}$ (to be more precise, $\chi_{0}$ is given by $\left.(\zeta I-\mathcal{A})^{-1} \mathcal{B} \widehat{w}_{0}\right)$ such that this EMPsignal input $w$ together with the initial state $\chi_{0}$ yields exactly the above-constructed EMP-signal output $z$ over the entire nonnegative time interval $[0, \infty)$. Conversely, it is also easy to show that if under some initial state $\chi_{0}$, the output $z$ of $\Sigma_{0}$ to some EMP-signal input $w$ with characteristic multiplier $\zeta$ is exactly an EMP-signal with the same characteristic multiplier over the whole nonnegative time interval $[0, \infty)$, then $\widehat{w}_{0}=\operatorname{INI}(w)$ and $\widehat{z}_{0}=\operatorname{INI}(z)$ are related by $(14)$.

Now, let us introduce the following definition.

DEFINITION 1. The EMP-signals $w$ and $z$ with the same characteristic multiplier are said to be consistent with the sampled-data system $\Sigma_{0}$ if there exists an initial state 
$\chi_{0}$ of $\Sigma_{0}$ such that the input $w$ yields exactly the output $z$ over the entire nonnegative time interval $[0, \infty)$.

Then, the above arguments can be summarized as follows.

Lemma 2. Suppose that $\zeta \notin \lambda(\mathcal{A})$. The relation (14) holds if and only if the EMP-signals $w=\operatorname{EMP}\left(\widehat{w}_{0}\right)$ and $z=\operatorname{EMP}\left(\widehat{z}_{0}\right)$ with characteristic multiplier $\zeta$ are consistent with the sampled-data system $\Sigma_{0}$.

3. Characterization of the spectrum of the transfer operator. The purpose of this section is to give a method for determining the spectrum of $\widehat{G}(\zeta)$ for $\zeta \notin \lambda(\mathcal{A})$. To give such a method, it is helpful to begin by studying some spectral properties of $\widehat{G}(\zeta)$. This is done in subsection 3.1, while in subsection 3.2 we give a method to determine $\sigma(\widehat{G}(\zeta))$.

3.1. Preliminary considerations on the spectrum. Let $\lambda\left(D_{11}\right)$ denote the set of the eigenvalues of the matrix $D_{11}$. Then, it is easy to show that

$$
\begin{aligned}
\sigma_{l e}(\widehat{G}(\zeta)) & =\sigma_{r e}(\widehat{G}(\zeta))=\sigma_{e}(\widehat{G}(\zeta))=\sigma_{l e}\left(D_{11}\right) \\
& =\sigma_{r e}\left(D_{11}\right)=\sigma_{e}\left(D_{11}\right)=\sigma\left(D_{11}\right)=\lambda\left(D_{11}\right)
\end{aligned}
$$

(see, e.g., [22], in particular Proposition XI.4.2, and [26], in particular Corollary XXIII.2.5). Since the essential spectrum is a subset of the spectrum, it follows that $\lambda\left(D_{11}\right)$ is a subset of $\sigma(\widehat{G}(\zeta))$ for any $\zeta \notin \lambda(\mathcal{A})$. Hence, to find all the points in the spectrum of $\widehat{G}(\zeta)$, it is enough for us to construct a method to check if $\gamma \notin \lambda\left(D_{11}\right)$ is a point in the spectrum of $\widehat{G}(\zeta)$. Thus, we assume $\gamma \notin \lambda\left(D_{11}\right)$ without loss of generality.

Since $\mathcal{D}=\mathcal{D}_{0}+D_{11}$, we have

$$
\gamma I-\widehat{G}(\zeta)=\left(\gamma I-D_{11}\right)\left(I-\widehat{G}_{\gamma}(\zeta)\right),
$$

where

$$
\widehat{G}_{\gamma}(z):=\left(\gamma I-D_{11}\right)^{-1}\left(\mathcal{C}(z I-\mathcal{A})^{-1} \mathcal{B}+\mathcal{D}_{0}\right) .
$$

Hence, by (16), it is obvious that $\gamma I-\widehat{G}(\zeta)$ is invertible if and only if $I-\widehat{G}_{\gamma}(\zeta)$ is. Since $\widehat{G}_{\gamma}(\zeta)$ is a compact operator because $\mathcal{D}_{0}$ is, it follows that $\gamma \notin \lambda\left(D_{11}\right)$ is a point in the spectrum of $\widehat{G}(\zeta)$ if and only if $\widehat{G}_{\gamma}(\zeta)$ has an eigenvalue at 1 . This is an important step for the following discussion, while the following result will also be useful.

Lemma 3. If $\gamma_{1} \in \partial \sigma(\widehat{G}(\zeta))$ and $\gamma_{1} \notin \lambda\left(D_{11}\right)$, then $\gamma_{1}$ is an isolated point of $\sigma(\widehat{G}(\zeta))$. [22].

Proof. By (15), the assertion follows immediately from Theorem XI.6.8 of

Now, we are in a position to show the following result.

THEOREM 4. $\sigma(\widehat{G}(\zeta)) \backslash \sigma_{e}(\widehat{G}(\zeta))$ coincides with $\sigma_{p}(\widehat{G}(\zeta)) \backslash \sigma_{e}(\widehat{G}(\zeta))$, where $\sigma_{p}(\cdot)$ denotes the point spectrum (i.e., the set of the eigenvalues of an operator). Furthermore, every $\gamma \in \sigma_{p}(\widehat{G}(\zeta)) \backslash \sigma_{e}(\widehat{G}(\zeta))$ is an isolated point of $\sigma(\widehat{G}(\zeta))$, and has finite multiplicity.

Remark 3.1. The above assertion is well known for a compact operator and also for a normal operator ([22, Proposition XI.4.6]), but $\widehat{G}(\zeta)$ is generally noncompact and nonnormal. It is not hard to see that the assertion in particular implies that the accumulation points of $\sigma(\widehat{G}(\zeta))$ can exist only at $\sigma_{e}(\widehat{G}(\zeta))=\lambda\left(D_{11}\right)$, which consists 
of finitely many points, and that $\sigma(\widehat{G}(\zeta)) \backslash \sigma_{e}(\widehat{G}(\zeta))$ (and thus $\sigma(\widehat{G}(\zeta))$, too) is a countable set. Hence, this proposition suggests that the properties of the spectrum of $\widehat{G}(\zeta)$ are almost as amenable as that of a compact normal operator.

Proof of Theorem 4. Since $\sigma_{e}(\widehat{G}(\zeta))=\lambda\left(D_{11}\right)$, for the first assertion it is enough to show that $\gamma \notin \lambda\left(D_{11}\right)$ belongs to $\sigma(\widehat{G}(\zeta))$ only if it is an eigenvalue of $\widehat{G}(\zeta)$. To show this, suppose that $\gamma \notin \lambda\left(D_{11}\right)$ is a point in $\sigma(\widehat{G}(\zeta))$. Then, by the arguments preceding Lemma $3, \widehat{G}_{\gamma}(\zeta)$ has an eigenvalue at 1 . This implies that there exists some nonzero $\widehat{w} \in \mathcal{K}$ such that $\left(I-\widehat{G}_{\gamma}(\zeta)\right) \widehat{w}=0$. Hence, it follows from (16) that $(\gamma I-\widehat{G}(\zeta)) \widehat{w}=0$, which implies that $\gamma$ is an eigenvalue of $\widehat{G}(\zeta)$. This completes the proof for the first assertion.

As for the second assertion, it is a direct consequence from [22, Corollary XI.2.4] that $\gamma \in \sigma_{p}(\widehat{G}(\zeta)) \backslash \sigma_{e}(\widehat{G}(\zeta))$ (which we abbreviate as $\sigma_{p} \backslash \sigma_{e}$ in what follows) has finite multiplicity, since for $\gamma \notin \lambda\left(D_{11}\right)=\sigma_{e}(\widehat{G}(\zeta)), \gamma I-\widehat{G}(\zeta)$ is Fredholm [22]. Thus, it remains only to show that every $\gamma \in \sigma_{p} \backslash \sigma_{e}$ is an isolated point of $\sigma(\widehat{G}(\zeta))$. Let $\gamma \in \sigma_{p} \backslash \sigma_{e}$. If $\gamma \in \partial \sigma(\widehat{G}(\zeta))$, then the assertion follows immediately from Lemma 3 . If $\gamma \notin \partial \sigma(\widehat{G}(\zeta))$, on the other hand, then $\gamma \in \sigma(\widehat{G}(\zeta))$ is an interior point of $\sigma(\widehat{G}(\zeta))$, and thus there exists some $\varepsilon$-neighborhood of $\gamma$ contained in $\sigma(\widehat{G}(\zeta))$. This, together with the compactness of $\sigma(\widehat{G}(\zeta))$ means that we can take some number $\gamma_{1}$ such that $\gamma_{1} \in \partial \sigma(\widehat{G}(\zeta))$ and at the same time $\gamma_{1}$ is not an isolated point of $\sigma(\widehat{G}(\zeta))$, where such $\gamma_{1}$ can always be taken so that $\gamma_{1} \notin \lambda\left(D_{11}\right)$ since $\lambda\left(D_{11}\right)$ is only a finite set. This contradicts Lemma 3 , and hence $\gamma \notin \partial \sigma(\widehat{G}(\zeta))$ cannot occur. This completes the proof.

3.2. Reduction to a finite-dimensional eigenvalue problem. Theorem 4 tells us that in essence we have only to find the eigenvalues of the operator $\widehat{G}(\zeta)$ to determine its spectrum. The purpose of this subsection is to give a result with which we can characterize the eigenvalues of $\widehat{G}(\zeta)$ through a finite-dimensional eigenvalue problem, and this is facilitated by the "nonasymptotic" characterization of the transfer operator.

To this end, let us consider the closed-loop sampled-data system $\Sigma_{1 / \gamma}$ (i.e., $\Sigma_{\Delta}$ with $\Delta$ set to $\frac{1}{\gamma} I$ in Figure 2), where $\gamma$ is a nonzero complex number. Let $\zeta \notin \lambda(\mathcal{A})$, and suppose that the responses of $w$ and $z$ in this closed-loop sampled-data system under the input $p=0, q=0$ and some appropriate initial state $\chi_{0}=\left[x(0)^{T}, \xi_{0}^{T}\right]^{T}$ are exactly EMP-signals of characteristic multiplier $\zeta$ over the entire nonnegative time interval. Then, it follows from Lemma 2 that (14) holds for $\widehat{w}_{0}=\operatorname{INI}(w)$ and $\widehat{z}_{0}=\operatorname{INI}(z)$. On the other hand, from Figure 2 (recall that $\Delta=\frac{1}{\gamma} I$ ), it is obvious that $\widehat{w}_{0}=\frac{1}{\gamma} \widehat{z}_{0}$. Hence we are led to

$$
(\gamma I-\widehat{G}(\zeta)) \widehat{w}_{0}=0
$$

Thus, we can conclude that $\gamma$ is an eigenvalue of $\widehat{G}(\zeta)$ if $\widehat{w}_{0} \neq 0$. This suggests that we can determine the eigenvalues of the transfer operator of the open-loop sampled-data system $\Sigma_{0}$ by considering the responses of the closed-loop sampled-data system $\Sigma_{1 / \gamma}$.

Now, when $q=0$, the continuous-time part of $\Sigma_{1 / \gamma}$ is described by

$$
\frac{d x}{d t}=A_{\gamma} x+B_{1 \gamma} p+B_{2 \gamma} u, \quad z=C_{1 \gamma} x+D_{11 \gamma} p+D_{12 \gamma} u, \quad y=C_{2} x,
$$


where

$A_{\gamma}:=A+B_{1}\left(\gamma I-D_{11}\right)^{-1} C_{1}, \quad B_{1 \gamma}:=\gamma B_{1}\left(\gamma I-D_{11}\right)^{-1}, \quad B_{2 \gamma}:=B_{2}+B_{1}\left(\gamma I-D_{11}\right)^{-1} D_{12}$,

$C_{1 \gamma}:=\gamma\left(\gamma I-D_{11}\right)^{-1} C_{1}, \quad D_{11 \gamma}:=\gamma\left(\gamma I-D_{11}\right)^{-1} D_{11}, \quad D_{12 \gamma}:=\gamma\left(\gamma I-D_{11}\right)^{-1} D_{12}$.

Hence, by also letting $p=0$, the lifted description of this closed-loop "autonomous" sampled-data system $\Sigma_{1 / \gamma}$ (i.e., without an external input) is given by

$$
\chi_{k+1}=\mathcal{A}_{\gamma} \chi_{k}, \quad \widehat{z}_{k}=\mathcal{C}_{\gamma} \chi_{k},
$$

where $\mathcal{A}_{\gamma}$ and $\mathcal{C}_{\gamma}$ are, respectively, given by $\mathcal{A}$ and $\mathcal{C}$ in (10) with $A, B_{2}, C_{1}$, and $D_{12}$ replaced by $A_{\gamma}, B_{2 \gamma}, C_{1 \gamma}$, and $D_{12 \gamma}$ in (20), respectively. Note that $\mathcal{A}_{\gamma}$ is nothing but the state transition matrix of the "discrete-time equivalent" of $\Sigma_{1 / \gamma}$.

We are in a position to state the following theorem.

THEOREM 5. Given a complex number $\gamma \notin \lambda\left(D_{11}\right)$ and a complex number $\zeta \notin$ $\lambda(\mathcal{A})$, the operator $\widehat{G}(\zeta)$ has an eigenvalue at $\gamma$ if and only if $\zeta I-\mathcal{A}_{\gamma}$ is not invertible.

Proof. We first establish the assertion assuming $\gamma \neq 0$. Let us first prove the sufficiency. Suppose that $\zeta I-\mathcal{A}_{\gamma}$ is not invertible.

Then, by the first equation in (21), the system $\Sigma_{1 / \gamma}$ has a nontrivial solution of the form

$$
\chi_{k}=\chi_{0} \zeta^{k}
$$

for some nonzero initial state $\chi_{0}$. Hence, by the second equation in (21), we can see that $z$ is an EMP-signal with characteristic multiplier $\zeta$. Since $w=\frac{1}{\gamma} z$, it follows that $w$ is also an EMP-signal with the same characteristic multiplier (carefully note that $w$ and $z$ could be both zero at this stage of our discussion). Thus, by the arguments preceding this theorem, we are led to (18). Therefore, it remains only to show that $\widehat{w}_{0} \neq 0$. To show this, suppose the contrary. Then, $w=0$ so that $\Sigma_{1 / \gamma}$ is essentially nothing but $\Sigma_{0}$ with $w=0$. Then, the existence of the nontrivial solution (22) contradicts the assumption that $\zeta \notin \lambda(\mathcal{A})$.

To prove the necessity, suppose that $\widehat{G}(\zeta) \widehat{w}_{0}=\gamma \widehat{w}_{0}$ for some $\widehat{w}_{0} \neq 0$. Then, letting $\widehat{z}_{0}:=\gamma \widehat{w}_{0}$, Lemma 2 implies that the EMP-signals $w=\operatorname{EMP}\left(\widehat{w}_{0}\right)$ and $z=$ $\operatorname{EMP}\left(\widehat{z}_{0}\right)=\gamma w$ with characteristic multiplier $\zeta$ are consistent with the open-loop sampled-data system $\Sigma_{0}$, so that these two EMP-signals can be represented as the responses of $w$ and $z$ in the closed-loop autonomous (i.e., $p=0, q=0$ ) system $\Sigma_{1 / \gamma}$ for some appropriate initial state $\chi_{0}$. Furthermore, since $w \neq 0$ is an EMP-signal, it follows readily that the discrete-time signal $\widehat{w}_{k}(\theta)$ is represented as $\widehat{w}_{0}(\theta) \zeta^{k}$, which is not identically zero as a sequence in $k$ at least for some $\theta \in[0, h)$ (note that the "sampling" of $\widehat{w}_{k}(\cdot)$ at $\theta$ is well defined since the signal $w$ is well behaved as a response of the autonomous sampled-data system $\Sigma_{1 / \gamma}$ ). Thus, from a basic property of discrete-time systems, it must be true that $\zeta$ is an eigenvalue of the transition matrix of the discrete-time equivalent of $\Sigma_{1 / \gamma}$ viewed at every sampling period $h$, which is given by $\mathcal{A}_{\gamma}$. This completes the proof for the case of $\gamma \neq 0$.

Finally, let us consider the case of $\gamma=0$ (note that $D_{11}$ is invertible in this case by the assumption $\left.\gamma \notin \lambda\left(D_{11}\right)\right)$. In this case, it is enough to consider $\widehat{G}(\zeta)+\alpha I(\alpha \neq 0)$ and study the condition for it to have an eigenvalue at $\alpha$. Noting that considering $\widehat{G}(\zeta)+\alpha I$ instead of $\widehat{G}(\zeta)$ is nothing but replacing $D_{11}$ with $D_{11}+\alpha I$ (for which 
$\left.\alpha \notin \lambda\left(D_{11}+\alpha I\right)\right)$, and observing the form of $A_{\gamma}$ and $B_{2 \gamma}$ given in (20), it is easy to see that the statement is valid even when $\gamma=0$.

Summarizing the arguments in this section, it follows that Theorems 4 and 5, together with (15), give a method to determine the spectrum of $\widehat{G}(\zeta)$ for each $\zeta \notin$ $\lambda(\mathcal{A})$. That is, every point in $\lambda\left(D_{11}\right)$ belongs to $\sigma(\widehat{G}(\zeta))$, and the remaining points in the spectrum can be found by searching for $\gamma$ such that $\zeta I-\mathcal{A}_{\gamma}$ is not invertible ${ }^{2}$. Since the spectral radius of $\widehat{G}(\zeta)$ is no larger than $\|\widehat{G}(\zeta)\|$, it is enough to consider the disk $\{\gamma:|\gamma| \leq\|\widehat{G}(\zeta)\|\}$ in such a search. An easily computable upper bound for $\|\widehat{G}(\zeta)\|$ can be obtained by an obvious extension of Theorem 1 of [27] (i.e., this theorem holds even if $|\zeta| \neq 1$ ) when $D_{11}=0$; if $D_{11} \neq 0$, a simple upper bound is obtained from a triangle inequality in which the upper bound is increased by $\left\|D_{11}\right\|$. These considerations give a basis for the numerical computation of $\sigma(\widehat{G}(\zeta))$, but we do not pursue numerical studies in this paper. Instead, we advance our study to demonstrate the importance of our spectral analysis for theoretical studies such as the stability and robust stability problems of sampled-data systems.

Remark 3.2. We point out that most of the discussions in this section carries over, without essential difficulties, to the case where the generalized plant $P$ is a finite-dimensional linear continuous-time $h$-periodic (FDLCP) system and $\Delta$ is an internally stable FDLCP system, where $h$ is the sampling period; the only nontrivial point will be the treatment of the essential spectrum. However, it is not hard to see that (15) still holds with $\lambda\left(D_{11}\right)$ replaced by

$$
\lambda_{[0, h]}\left(D_{11}\right):=\left\{\lambda \mid \text { the set of } t \in[0, h] \text { such that }\left|\operatorname{det}\left(\lambda I-D_{11}(t)\right)\right|<\gamma\right. \text { has }
$$

$$
\text { nonzero measure whenever } \gamma>0\} \text {, }
$$

which follows from section XXIII.2 of [26]. Thus, the arguments in this section still apply mutatis mutandis. The only point that requires some more careful arguments will be the isolatedness assertion in Theorem 4 , since $\sigma_{e}(\widehat{G}(\zeta))=\lambda_{[0, h]}\left(D_{11}\right)$ can now form a closed curve; thus the arguments in the proof of Theorem 4 are not enough to establish the isolatedness of some eigenvalues within the essential spectrum radius. Fortunately, however, the isolatedness property is not relevant for Theorem 5, which will be used as a major tool in the following section which demonstrates the usefulness of the spectral analysis in this section.

4. Application to robust internal stability problems. In $[16,17]$, the positive-realness notion was introduced to sampled-data systems and some phase properties of sampled-data systems were also addressed. A more advanced study on the positive-realness of sampled-data systems was pursued, and the positive-realness gap index $\rho_{\min }$ was introduced in $[18,19]$. It was also shown in [19] that this index plays an important role in the positive-realness approach (or the passivity approach) to the stability analysis of sampled-data systems. In this section, we first review the above-mentioned study in [19] briefly, and then show that our study in the preceding sections has an important application to such or more general stability and robust stability analysis.

In subsection 4.1 , we deal with the gain margin analysis problem of sampleddata systems and derive some useful results by applying the spectral analysis in the

\footnotetext{
${ }^{2}$ It would be possible to determine the multiplicity of $\gamma$ as an eigenvalue of $\widehat{G}(\zeta)$ by considering the geometric multiplicity of $\zeta$ as an eigenvalue of $\zeta I-\mathcal{A}_{\gamma}$, if we introduce some sort of controllability/observability conditions. However, we do not pursue this direction in this paper.
} 
preceding section (in particular, Theorem 5). Then, such results will be applied in subsection 4.2 to give a result about robust stability of sampled-data systems.

4.1. Gain margin analysis for internal stability in the sampled-data context. The transfer operator $\widehat{G}(z)$ of the internally stable sampled-data system $\Sigma_{0}$ is said to be strongly positive-real [19] if there exists a positive number $\varepsilon$ such that

$$
\widehat{G}(z)+\widehat{G}(z)^{*} \geq \varepsilon I \quad(\forall|z| \geq 1) .
$$

The transfer operator $\widehat{G}(z)$ is not strongly positive-real, in general, but we can consider the following number:

$$
\rho_{\min }:=\inf _{\rho>0}\{\widehat{G}(z)+\rho I \text { is strongly positive-real }\} \geq 0 .
$$

This number is called the positive-realness gap index for $\Sigma_{0}$, and plays an important role in the stability analysis as shown in [19]; one important result shown about this index is that for $k>0$, the (negative feedback) closed-loop sampled-data system $\Sigma_{-k}$ (i.e., $\Sigma_{\Delta}$ with $\Delta$ set to $-k I$ ), or to be more precise, the input-output mapping $\mathcal{G}_{-k}$ is $L_{2}$-stable if $0<k<k_{\max }^{\mathrm{PR}}$, where

$$
k_{\max }^{\mathrm{PR}}:=1 / \rho_{\min } .
$$

By a suitable construction of the generalized plant $P$, this "gain margin analysis problem" in the context of sampled-data control can represent a sort of stabilityradius analysis problem with respect to the uncertainties in the physical parameters of the plant, and as such, to compute $k_{\max }^{\mathrm{PR}}$ is quite important. In [19], an efficient finite-dimensional state-space method for the computation of $\rho_{\min }$ and thus $k_{\max }^{\mathrm{PR}}$ was given. Furthermore, an iterative procedure was given to compute the number $k_{\max }\left(\geq k_{\max }^{\mathrm{PR}}\right)$, which is defined as the largest $\bar{k}$ such that $\Sigma_{-k}(k>0)$ is internally stable for all $k<\bar{k}$. However, in the derivation of that procedure, the following result was used without proof; we now give its proof by applying the spectral analysis results in the preceding section so that the procedure for computing $k_{\max }$ given in [19] is validated rigorously.

Proposition 6. If $\Sigma_{0}$ is internally stable, then $\Sigma_{-k}$ is internally stable for all $k \in\left(0, k_{\max }^{\mathrm{PR}}\right)$.

Proof. Now, suppose the contrary. Then, by the definition of internal stability $[13,23]$, there exists some $k^{\star} \in\left(0, k_{\max }^{\mathrm{PR}}\right)$ such that the state transition matrix of $\Sigma_{-k^{\star}}$ has an eigenvalue on or outside the unit circle, say at $\zeta^{\star}$. Therefore, it follows from the definition of $\mathcal{A}_{\gamma}$ that if we put $-\rho^{\star}:=-1 / k^{\star}$, then $\zeta^{\star} I-\mathcal{A}_{-\rho^{\star}}$ is not invertible where $\left|\zeta^{\star}\right| \geq 1$. Here, note that

$$
\rho^{\star}>\rho_{\min } \geq 0
$$

since $0<k^{\star}<k_{\max }^{\mathrm{PR}}$ by the assumption. Thus, by the properties of strongly positivereal transfer operators [19], we have $D_{11}+D_{11}^{T}+2 \rho^{\star} I>0$. This in particular implies that $-\rho^{\star} \notin \lambda\left(D_{11}\right)$. Summarizing the above arguments and applying Theorem 5, we are led to the conclusion that $\widehat{G}\left(\zeta^{\star}\right)$ has an eigenvalue at $-\rho^{\star}$. This in particular implies that $\widehat{G}\left(\zeta^{\star}\right)+\widehat{G}\left(\zeta^{\star}\right)^{*}+2 \rho^{\star} I \ngtr 0$. Since $\left|\zeta^{\star}\right| \geq 1$, it follows that $\widehat{G}(z)+\rho^{\star} I$ is not a strongly positive-real transfer operator, and hence $\rho^{\star} \leq \rho_{\min }$. This clearly contradicts (27). Hence, we have established that $\Sigma_{-k}$ is internally stable for all $k \in\left(0, k_{\max }^{\mathrm{PR}}\right)$. 
It would be worthwhile mentioning that the above proposition can be extended to $\Sigma_{k}(k>0)$ by considering $-\widehat{G}(z)$ instead and redefining $\rho_{\min }$ and thus $k_{\max }^{\mathrm{PR}}$ accordingly.

Next, we claim Proposition 7 given below, which plays a crucial role in the robust stability analysis in the following subsection.

Proposition 7. Suppose that $\Sigma_{0}$ is internally stable. Then, for each fixed $k, \Sigma_{k}$ is internally stable if and only if $\mathcal{G}_{k}$ is $L_{2}$-stable.

Remark 4.1. This proposition does not say that an (open-loop) sampled-data system is internally stable if and only if it is $L_{2}$-stable, an assertion (the sufficiency part) that is obviously false; note that this proposition deals only with such (closedloop) sampled-data systems that can arise from an internally stable sampled-data system. Note also that the condition $1 / k \notin \lambda\left(D_{11}\right)$, which is the well-posedness condition of $\Sigma_{k}$ and at the same time the well-definedness condition for $\mathcal{G}_{k}$, is implicit in the proposition. To be more precise, if we say that $\Sigma_{k}$ is internally stable or $L_{2}$ stable, it in particular means that $1 / k \notin \lambda\left(D_{11}\right)$. Further, note that this proposition shows in particular that $k_{\max , L_{2}}=k_{\max }$, where $k_{\max , L_{2}}$ denotes the largest number $\bar{k}$ such that $\mathcal{G}_{k}$ is $L_{2}$-stable whenever $0<k<\bar{k}$, while $k_{\max }$ has been defined similarly but in terms of internal stability. It should be stressed, however, that the assertion of the above proposition holds even if $k>k_{\max }$, as long as $\Sigma_{k}$ or $\mathcal{G}_{k}$ is stable for such $k$.

Proof of Proposition 7. Let us begin with the necessity part. It is not hard to see by the inspection of the procedure for computing $k_{\max }$ given in [19] that the set of $k$ for which $\Sigma_{k}$ is internally stable is a subset of those $k$ for which $\Sigma_{k}$ is $L_{2^{-}}$ stable $^{3}$. Hence, the necessity assertion follows immediately. (Instead of this proof that is based on the transfer operator $\widehat{G}(z)$ and the spectral analysis results in the preceding section as a whole, an alternative proof is also possible in which we use the well-known fact that an internally stable sampled-data system is $L_{2}$-stable under mild conditions [13, 23].)

To show the sufficiency part, we assume that $\Sigma_{k}$ is not internally stable for some $k$ (i.e., $\zeta I-\mathcal{A}_{\gamma}$ with $\gamma:=1 / k$ is not invertible for some $|\zeta| \geq 1$ ), and show that $\mathcal{G}_{k}$ is not $L_{2}$-stable. Here, it is enough to assume $1 / k \notin \lambda\left(D_{11}\right)$, because otherwise $\mathcal{G}_{k}$ is not $L_{2}$-stable as stated in Remark 4.1.

Since $\zeta \notin \lambda(\mathcal{A})$ by the internal stability assumption of $\Sigma_{0}$, it follows from Theorem 5 that $\widehat{G}(\zeta) \widehat{w}_{0}=\gamma \widehat{w}_{0}$ for some $\widehat{w}_{0} \neq 0$. Hence by Lemma 2 , there exists an appropriate initial state $\chi_{0}=\chi^{\star}$ of $\Sigma_{0}$ such that $w=\operatorname{EMP}\left(\widehat{w}_{0}\right)$ and $z=\gamma \operatorname{EMP}\left(\widehat{w}_{0}\right)$ are consistent with $\Sigma_{0}$ (the corresponding characteristic multiplier for the EMP signals is $\zeta$ throughout the proof). Now, let us denote by $z^{\star}$ the response of $z$ in $\Sigma_{0}$ when its initial state is $\chi_{0}=\chi^{\star}$ and its input is $w=0$. Note that $z^{\star} \in L_{2}$ by the internal stability of $\Sigma_{0}$, which is well known, e.g., in the context of sampled-data $H_{2}$ problem [23]. Also, by linearity, it follows immediately that $\Sigma_{0}$ yields the response $z=\gamma \operatorname{EMP}\left(\widehat{w}_{0}\right)-z^{\star}$ when the initial state is $\chi_{0}=0$ and the input is $w=\operatorname{EMP}\left(\widehat{w}_{0}\right)$. Hence, it is easy to see that $\Sigma_{\Delta}$ with $\Delta=(1 / \gamma) I$ yields $f=w=\operatorname{EMP}\left(\widehat{w}_{0}\right), z=\gamma \operatorname{EMP}\left(\widehat{w}_{0}\right)-z^{\star}$, and $e=\gamma \operatorname{EMP}\left(\widehat{w}_{0}\right)$ when the initial state is $\chi_{0}=0$ and the inputs are $p=0 \in L_{2}$ and

\footnotetext{
${ }^{3}$ In a sense, a direct application of the arguments in [19] is limited only to $k \in\left[0, k_{\text {max }}\right]$. However, if $\Sigma_{k}$ is internally stable for some $k=k^{\star} \notin\left[0, k_{\max }\right]$, then we can readily introduce a modified generalized plant $P_{k^{\star}}$ such that $\Sigma_{k}$ can be viewed as $\Sigma_{k^{\prime}}^{\star}$ (and thus $\Sigma_{k^{\star}}$ can be viewed as the openloop sampled-data system $\Sigma_{0}^{\star}$ ), where $k^{\prime}=k-k^{\star}$ and $\Sigma_{k}^{\star}$ denotes $\Sigma_{k}$ with $P$ replaced by $P_{k^{\star}}$ (this idea of introducing a modified generalized plant is quite similar to that employed in the procedure for computing $k_{\max }$; see [19] for details). Hence, we can repeat the same arguments on $\Sigma_{k}^{\star}$, which implies that the assertion can be established even for those $k$ around $k^{\star} \notin\left[0, k_{\max }\right]$.
} 
$q=z^{\star} \in L_{2}$. Noting that none of these $f, w, z$, and $e$ belong to $L_{2}$ since $|\zeta| \geq 1$, we can conclude that $\mathcal{G}_{k}$ is not $L_{2}$-stable.

Remark 4.2. Even though it can be seen that Proposition 6 is implied by Proposition 7, it should be noted that the independent proof of Proposition 6 is indispensable. This is because in the proof of Proposition 7, we have referred to the procedure for computing $k_{\max }$ stated in [19], which in turn has been validated rigorously by the very proof of Proposition 6 .

4.2. Robust internal stability of sampled-data systems. Now, we are in a position to demonstrate the significance of Proposition 7, not merely in justifying the arguments of [19] about the computation of $k_{\max }$ through Proposition 6. More specifically, we give the following theorem about robust stability of the sampled-data system $\Sigma_{\Delta}$, which clarifies the relationship between robust $L_{2}$-stability and robust internal (exponential) stability.

THEOREM 8. Consider the closed-loop sampled-data system $\Sigma_{\Delta}$ shown in Figure 2 , where we assume that $D_{11}$ is possibly nonsquare and $\Delta \in \boldsymbol{\Delta}$ for some set $\boldsymbol{\Delta}$ of (possibly nonsquare) finite-dimensional linear time-invariant (FDLTI) internally stable systems, and suppose that $\Sigma_{0}$ is internally stable. Then, $\Sigma_{\Delta}$ is internally stable for all $\Delta \in \boldsymbol{\Delta}$ if and only if $\mathcal{G}_{\Delta}$ is $L_{2}$-stable for all $\Delta \in \boldsymbol{\Delta}$.

Proof. The assertion is almost just a direct consequence from Proposition 7 , but we need some careful arguments.

Let us begin with the sufficiency proof. Consider the (series-connected) open-loop sampled-data system $\Sigma_{0} \Delta$, and observe that it can be represented as the open-loop sampled-data system shown in Figure 1 with the generalized plant $P$ replaced by $P_{\Delta}:=P \operatorname{diag}[\Delta, I]$ (for which the " $D_{11}$ matrix" is square and thus the preceding arguments apply). Also, by the internal stability assumptions, $\Sigma_{0} \Delta$ is internally stable. Thus, this reformation corresponds to replacing $\Sigma_{0}$ and $\Delta$ by $\Sigma_{0} \Delta$ and 1 , respectively, if we interpret it in the closed-loop sampled-data system in Figure 2; let us denote by $\mathcal{G}_{\Delta}^{\prime}$ the corresponding input-output mapping of thus reformed closedloop sampled-data system. Since $\mathcal{G}_{\Delta}$ is $L_{2}$-stable for each $\Delta \in \boldsymbol{\Delta}$, it is straightforward to show that $\mathcal{G}_{\Delta}^{\prime}$ is also $L_{2}$-stable for each $\Delta \in \boldsymbol{\Delta}$. Thus, applying Proposition 7 with $k=1$ leads to the assertion.

To show the necessity, we also consider the closed-loop sampled-data system $\Sigma_{\Delta}$ with $\Sigma_{0}$ and $\Delta$ replaced by $\Delta \Sigma_{0}$ and 1 , respectively, and denote by $\mathcal{G}_{\Delta}^{\prime \prime}$ the corresponding input-output mapping. Applying Proposition 7 with $k=1$, it follows readily that both $\mathcal{G}_{\Delta}^{\prime}$ and $\mathcal{G}_{\Delta}^{\prime \prime}$ are $L_{2}$-stable. In view of the linearity of $\Sigma_{0}$ and $\Delta$ (and thus the mapping $\mathcal{G}_{\Delta}$ ), it is not hard to show that $L_{2}$-stability of $\mathcal{G}_{\Delta}^{\prime}$ and $\mathcal{G}_{\Delta}^{\prime \prime}$ implies that of $\mathcal{G}_{\Delta}$.

This completes the proof.

Remark 4.3. If we recall Remark 3.2, it is not hard to see that Proposition 7 and Theorem 8 still hold even when $P$ (and/or $\Delta$ ) is a finite-dimensional linear continuoustime $h$-periodic system. Also, concerning Figure 2, a typical interpretation is that $P_{22}$ (the subsystem from $u$ to $y$ ) denotes the nominal plant, while the perturbed (actual) plant is represented by the upper LFT (linear fractional transformation) $\mathcal{F}_{u}(P, \Delta)=$ : $P_{22 \Delta}$. Since uncontrollable/unobservable modes do not affect internal (exponential) stability if and only if they are stable, this theorem in particular says that

(i) any robust stability condition such as the small-gain condition in particular guarantees that no unstable pole/zero cancellations can occur within $P_{22 \Delta}$, irrespectively of $\Delta$ belonging to the perturbation set $\boldsymbol{\Delta}$ that the condition takes care of, 
and it is obvious that

(ii) the discrete-time controller $\Psi$ internally (exponentially) stabilizes any stabilizable detectable plants whose minimal realization coincides with that of $P_{22 \Delta}$ for some $\Delta$ in the corresponding $\Delta$.

In particular, (ii) implies that $\Psi$ could internally stabilize also a lower-order plant than the generalized plant (because of stable pole/zero cancellations via $\Delta$ ), even though this might not be necessarily clear in the above arguments where we treated $\Delta$ in such a way that it has an independent state from the nominal plant.

One important perturbation set $\boldsymbol{\Delta}$ is the set of norm-bounded LTI perturbations, for which the small-gain condition can be very conservative $[6,8]$. To get around such conservatism, a necessary and sufficient condition has been derived $[6,8]$ in the $L_{2}$-stability context. The above theorem guarantees that such a necessary and sufficient condition is indeed necessary and sufficient for robust internal stability; this has already been shown for special perturbations (i.e., additive and multiplicative perturbations), including the case of unstable perturbations [9]. The above theorem in particular extends the previous results to the case of general perturbation structures but with stable perturbations.

To state the importance of this theorem more generally and precisely, we can rephrase it in the following way: whatever allowable perturbation sets/structures we may consider (for example, LTI/ $h$-periodic perturbations, full-block/block-diagonal structures, real-parametric/dynamical perturbations, norm-bounded/unbounded perturbations, connected/nonconnected perturbation sets, convex/nonconvex perturbations with respect to the origin ${ }^{4}$, and so on, as well as their arbitrary combinations), considering robust $L_{2}$-stability is enough to ensure robust internal stability; once a condition for robust $L_{2}$-stability under such FDLTI stable perturbations is established somehow, the condition automatically guarantees robust internal stability. It would also be worth stressing that the theorem applies even to such cases where some part of the perturbations are fictitious and introduced just for the robust performance analysis/synthesis (so that they do not affect internal stability) while the remaining part of the perturbations does represent the plant uncertainty, as in the main loop theorem. Robust internal stability is obviously guaranteed by robust $L_{2}$-stability even in such cases with robust performance taken into consideration.

5. Conclusion. In this paper, we first gave a nonasymptotic characterization of the transfer operator $\widehat{G}(z)$ of sampled-data systems, so that some appropriate nonzero initial states can be introduced into the study of the transfer operator $\widehat{G}(z)$. Based on such a characterization, we then studied the spectral properties of the transfer operator $\widehat{G}(z)$. More specifically, it was shown that the properties of the spectrum of $\widehat{G}(z)$ are nearly as amenable as those of compact normal operators, in spite of the generally noncompact and nonnormal nature of $\widehat{G}(z)$, and that the spectrum can be characterized with finite-dimensional eigenvalue problems. Exploiting a close relation with the eigenvalue problems and the condition for internal stability of sampled-data systems, we further extended our arguments on the spectral analysis of $\widehat{G}(z)$ to the robust internal stability analysis of sampled-data systems. To summarize the results very concisely, what we have shown is that robust $L_{2}$-stability and robust internal (exponential) stability are equivalent irrespective of the perturbation structures/sets to be considered, if the nominal sampled-data system is internally stable, if the

\footnotetext{
${ }^{4}$ We say here that $\boldsymbol{\Delta}$ is convex with respect to the origin if $\Delta \in \boldsymbol{\Delta}$ implies $k \Delta \in \boldsymbol{\Delta}$ for all $k \in[0,1]$.
} 
perturbations are either finite-dimensional LTI or $h$-periodic, and if the perturbations are internally stable. Although we confined our input-output stability notion to $L_{2}$-stability in this paper, it is not hard to see that Proposition 7 and thus Theorem 8 hold even if $L_{2}$-stability is replaced by $L_{p}$-stability, where $1 \leq p<\infty$. Hence, a solid theoretical basis is established for the robust stabilization/performance design for sampled-data systems even when it is carried out only under such input-output stability conditions as in [6]. Finally, it will be worthwhile mentioning that all the results in section 4 can be specialized to the continuous-time setting without any changes (since a continuous-time system can always be embedded into the class of sampled-data systems), and can readily be generalized to the discrete-time setting.

\section{REFERENCES}

[1] B. A. Bamieh And J. B. Pearson, A general framework for linear periodic systems with applications to $H_{\infty}$ sampled-data control, IEEE Trans. Automat. Control, 37 (1992), pp. 418-435.

[2] H. T. Tolvonen, Sampled-data control of continuous-time systems with an $H_{\infty}$ optimality criterion, Automatica J. IFAC, 28 (1992), pp. 45-54.

[3] P. T. Kabamba And S. HARA, Worst-case analysis and design of sampled-data control systems, IEEE Trans. Automat. Control, 38 (1993), pp. 1337-1357.

[4] Y. Hayakawa, Y. Yamamoto, And S. HaRA, $H_{\infty}$ type problem for sampled-data control systems - A solution via minimum energy characterization, IEEE Trans. Automat. Control, 39 (1994), pp. 2278-2284.

[5] N. Sivashankar And P. P. Khargonekar, Robust stability and performance analysis of sampled-data systems, IEEE Trans. Automat. Control, 38 (1993), pp. 58-69.

[6] G. Dullerud And K. Glover, Robust stabilization of sampled-data systems to structured LTI perturbations, IEEE Trans. Automat. Control, 38 (1993), pp. 1497-1508.

[7] G. E. Dullerud AND K. Glover, Analysis of structured LTI uncertainty in sampled-data systems, Automatica J. IFAC, 31 (1995), pp. 99-113.

[8] Y. OIsHI, Computation-oriented expression of a non-conservative condition for robust stability of sampled-data systems, Internat J. Control, 62 (1995), pp. 1085-1104.

[9] T. Hagiwara AND M. ARAKI, Robust stability of sampled-data systems under possibly unstable additive/multiplicative perturbations, IEEE Trans. Automat. Control, 43 (1998), pp. 13401346.

[10] Y. Yамамото, A function space approach to sampled data systems and tracking problems, IEEE Trans. Automat. Control, 39 (1994), pp. 703-713.

[11] Y. Yamamoto and P. P. Khargonekar, Frequency response of sampled-data systems, IEEE Trans. Automat. Control, 41 (1996), pp. 166-176.

[12] M. Araki, Y. Ito, And T. Hagiwara, Frequency response of sampled-data systems, Automatica J. IFAC, 32 (1996), pp. 483-497.

[13] T. Chen And B. A. Francis, Input-output stability of sampled-data systems, IEEE Trans. Automat. Control, 36 (1991), pp. 50-58.

[14] T. Chen And B. Francis, On the $L_{2}$-induced norm of a sampled-data system, Systems Control Lett. 15 (1990), pp. 211-219.

[15] Y. Үамамото, On the state space and frequency domain characterization of $H_{\infty}$-norm of sampled-data systems, Systems Control Lett. 21 (1993), pp. 163-172.

[16] K. Sugimoto and M. Suzuki, On $\gamma$-positive real sampled-data control systems, Proceedings of the 13th International Symposium on Mathematical Theory of Networks and Systems, Padova, Italy, 1998, pp. 409-412.

[17] K. Sugimoto And M. Suzuki, On $\gamma$-positive real sampled-data control systems and their phase property, (in Japanese), Transactions of the Society of Instrument and Control Engineers, 35 (1999), pp. 71-76.

[18] T. Hagiwara, Nyquist stability criterion and positive-realness of sampled-data systems, Systems Control Lett. 45 (2002), pp. 283-291.

[19] T. Hagiwara And T. Mugiuda, Positive-realness analysis of sampled-data systems and its applications, Automatica J. IFAC, 40 (2004), pp. 1043-1051.

[20] Y. Yамамото and S. HaRA, Relationships between internal and external stability for infinitedimensional systems with applications to a servo problem, IEEE Trans. Automat. Control, 33 (1988), pp. 1044-1052. 
[21] Y. YAмамото AND S. HARA, Internal and external stability and robust stability condition for a class of infinite-dimensional systems, Automatica J. IFAC, 28 (1992), pp. 81-93.

[22] J. B. Conway, A Course in Functional Analysis, 2nd ed., Springer-Verlag, New York, 1990.

[23] T. Chen and B. Francis, Optimal Sampled-Data Control Systems, Springer-Verlag, Berlin, 1995.

[24] T. HAGIWARA, Spectral analysis and singular value computations of the noncompact frequency response and compression operators in sampled-data systems, SIAM J. Control Optim., 41 (2002), pp. 1350-1371.

[25] N. M. Wereley, Analysis and Control of Linear Periodically Time Varying Systems, Ph.D. thesis, Dept. of Aeronautics and Astronautics, MIT, Cambridge, MA, 1990.

[26] I. Gohberg, S. Goldberg, and M. A. KaAshoek, Classes of Linear Operators, Vol. II, Birkhäuser, Basel, Switzerland, 1993.

[27] T. Hagiwara, M. Suyama, and M. Araki, Upper and lower bounds of the frequency response gain of sampled-data systems, Automatica J. IFAC, 37 (2001), pp. 1363-1370. 